\title{
Survival of the grouped, or three's a crowd? Repetition blindness in groups of letters and words
}

\author{
Andrea Jackson ${ }^{1}$ - Lori Buchanan ${ }^{1}$
}

Published online: 28 September 2015

(C) Psychonomic Society, Inc. 2015

\begin{abstract}
When stimuli are presented rapidly, repetitions are often undetected - a phenomenon called "repetition blindness" (RB; Kanwisher Cognition, 27, 117-143, 1987). Grouping of nonlinguistic items has been found to prevent RB (Goldfarb \& Treisman Psychonomic Bulletin \& Review, $18,1042-1049,2011)$. In order to determine whether this effect could be found with letters and words, participants viewed rapid serial visual presentation and brief simultaneous visual presentation streams containing groups of linguistic stimuli and provided judgments of frequency. The collection of reaction times and an explicit question about strategy use allowed for analyses of the participants' processing strategies. Two groups of participants emerged: one that demonstrated RB for groups of stimuli, and another that demonstrated enhanced perception with stimulus grouping. These participant groups did not appear to differ on the basis of explicit processing strategies or reaction times.
\end{abstract}

Keywords Repetition blindness - Survival of the grouped · Grouping $\cdot$ RSVP $\cdot$ BSVP

Repetition blindness (RB) is a reliable and robust phenomenon that operates at the interface of perception, semantics, and memory encoding. It is the failure to detect a repeated occurrence of a visual stimulus when items are presented at a rapid pace (Kanwisher, 1987). For example, in a presentation consisting of four letters (ABAC), participants will only report one occurrence of the letter A. The speed of presentation

Lori Buchanan

buchanan@uwindsor.ca

1 Psychology Department, University of Windsor, 401 Sunset Avenue, Windsor, Ontario N9B 3P4, Canada needed to achieve this effect is about $100-150 \mathrm{~ms}$ per item and can be attained through the use of either rapid serial visual presentation (RSVP; single items presented sequentially) or brief simultaneous visual presentation (BSVP; all items presented at once) (Kanwisher, 1991; Luo \& Caramazza, 1996).

$\mathrm{RB}$ is assumed to occur at the level of encoding (Epstein \& Kanwisher, 1999; Kanwisher, Kim, \& Wickens, 1996; Luo \& Caramazza, 1996; Neill, Neely, Hutchinson, Kahan, \& VerWys, 2002), prior to the convergence of auditory and visual inputs (Kanwisher \& Potter, 1989). This level of processing, though early, is still fairly abstract, since RB acts on general stimulus identity rather than strict visual form. For example, RB occurs even when letters or words differ in case (e.g., "sofa" and "SOFA"; Bavelier \& Potter, 1992; Kanwisher, 1987; Schendan, Kanwisher, \& Kutas, 1997) or in orientation (Coltheart, Mondy, \& Coltheart, 2005; Corballis \& Armstrong, 2007). Moreover, exact stimulus identity does not seem to be a requirement: RB has been found for a number of merely similar items, including those that share phonology (Bavelier, 1994; Bavelier \& Potter, 1992), orthography (Harris \& Morris, 1998, 2000, 2004; Morris \& Harris, 2002), or conceptual/semantic identity (Bavelier, 1994).

Multiple explanations of RB exist, but the most wellreceived is Kanwisher's (1987) token individuation hypothesis, in which the "blindness" is not for the items themselves, but for the distinction between the items; that is, the repeated items are not recognized as two discrete events. In this view, item perception involves the activation of a "type," or a representation in long-term memory, and the creation of a "token, " or a memory for that particular instance of the type. Under conditions that produce RB, an item's type is repeatedly activated, but the creation of multiple tokens ultimately fails (Kanwisher, 1987).

Bavelier and Potter (1992) extended this theory by positing that tokens are established via first the creation of a token and 
then the stabilization of that token in memory. Tokens for both presentations of the RB items may initially be created, but one is subsequently lost if it is not properly stabilized via registering information about the type (e.g., phonology, orthography) in memory.

The token individuation hypothesis (Kanwisher, 1987) provides an eloquent and thoughtful explanation of RB when considering two presentations of an item in an otherwise heterogeneous RSVP or BSVP display, as is the standard in the many RB studies. However, these heterogeneous displays typically have at least one nonidentical item inserted between repeated items. What if that were not the case? In Kanwisher's (1987) view, RB should occur for successive identical items, as the theory itself does not speak to the necessity of intervening items. Data consistent with this have come from Mozer (1989), who found that participants frequently underestimated strings of repeated letters. Specifically, Mozer investigated what Frick (1987) originally termed the "homogeneity effect" using frequency estimations of homogeneous versus heterogeneous strings of letters. Mozer suggested that consistent underestimation of homogeneous displays is the spatial analogue to the temporal effects Kanwisher (1987) theorized produce RB.

Goldfarb and Treisman (2011) reported results that differed from those of Mozer (1989) using simultaneous (BSVP) displays containing colored symbols. Trials were composed of either one repetition with an intervening item (e.g., ABAC format) or three identical grouped items (e.g., ABBB format). Quite expectedly, they observed an RB effect for the singlerepetition condition, but enhanced accuracy for the grouped condition. Coining this effect "the survival of the grouped," they suggested that their findings are still consistent with current theories of RB. Specifically, they proposed that the group of items is seen as a single, multifaceted item (akin to a face being composed of eyes, nose, mouth, etc.), as opposed to three individual units. As such, this group would require only a single episodic token (or "object file," an analogous concept; Goldfarb \& Treisman, 2011).

Further evidence for a "survival of the grouped" effect and support for Goldfarb and Treisman's (2011) suggestion that multiple items can become a meaningful single unit has come from Abrams, Dyer, and MacKay (1996). In their experiment, sentences were presented in RSVP in either syntactically correct parsings (e.g., "They wanted/to play sports/but sports/ were not allowed") or syntactically incorrect parsings (e.g., "They wanted to/play sports but/sports were not/allowed"). A repetition deficit for only the syntactically incorrect condition was hypothesized to be due to the ease of processing associated with syntactically correct grouping.

Goldfarb and Treisman (2011) suggested that the effect of grouping would not be as salient in RSVP as in BSVP, but prior research has suggested that tokens can extend across time as well as space (Kahneman \& Treisman, 1992). For example, in apparent motion, two carefully timed and spatially separated items appear to be a single, moving object-or a single episodic token (Kahneman \& Treisman, 1992). The creation of object files, as described by Kahneman and Treisman (1992) as well as by Kahneman and colleagues (1983), is guided by the unity and continuity of an item. Accordingly, it could be argued that traditional RB displays are distinctly disconnected, given the presence of an intervening item, but an uninterrupted display of three identical items would be likely to be perceived as a continuous, single unit.

Taken together, the evidence indicates that a preexisting suggestion of a group or larger unit (e.g., a syntactically correct phrase or same-colored forms) affords protection from RB (Abrams et al., 1996; Goldfarb \& Treisman, 2011). This is consistent with the idea that the unit on which RB operates is the unit that is attended by the participant (Kanwisher \& Potter, 1990). The phenomenon of apparent motion also supports the possibility of preserved perception of a group in a unified and continuous sequential display (Kahneman \& Treisman, 1992), but Mozer's (1989) underestimations of simultaneous displays of homogeneous letters appear to offer no such suggestion of a larger unit.

What remains unclear is whether this homogeneity effect is restricted to Mozer's (1989) original conditions (homogeneous items presented simultaneously), or whether it can be found in traditional RB experimental conditions, which include heterogeneous RSVP. Specifically, would a group of identical items appear more "group-like," or would the grouping be more salient if it was presented in the context of other items with which it could be contrasted (e.g., AABB) than in a string of identical items (e.g., AAAA)? If the homogeneity effect were found in traditional RB experimental conditions with linguistic stimuli, it would suggest that such "survival of the grouped" effects are limited to nonlinguistic stimuli or syntactically grouped linguistic stimuli.

In the present experiments, we aimed to determine whether Mozer's (1989) homogeneity effect can be found in traditional RB conditions (both letters and words, RSVP and BSVP displays) or whether under such conditions a "survival of the grouped" effect would be seen. In Experiments 1 and 2, we used RSVP and BSVP displays of single letters, and in Experiments 3 and 4 we investigated this phenomenon using the same types of displays with four-letter words. Experiment 5 was designed to clarify and replicate the results found in the initial experiments as well as further specific hypotheses about strategy.

The role of participant strategy in these tasks is of interest and was explored using a judgment-of-frequency (JOF) response coupled with reaction times (RTs). According to Brown, Buchanan, and Cabeza (2000), when JOFs are produced through a familiarity-based strategy (i.e., a "gut feeling"), RTs are relatively unchanged across conditions. Alternatively, when participants actively tally specific 
instances of a target item, the RT increases with the number of items presented. Although such an increase in RT was seen with Wong and Chen's (2009) work when participants performed an RB task in an RSVP format, the same effect has not been examined with grouped presentations.

In sum, the following experiments were designed to answer two questions: Under standard RB conditions using linguistic stimuli, (1) is a homogeneity effect or a "survival of the grouped" effect more likely? and (2) what strategy do viewers use to complete this task?

\section{Experiment 1}

Experiment 1 was designed to determine whether grouping or RB would result for letters in an RSVP format. Additionally, RTs were collected in order to determine the strategy used to complete this task.

\section{Method}

Participants Twenty University of Windsor undergraduate psychology students (18 female, two male; mean age $=$ 19.65 years, age range $=18-22$ ) participated. The numbers of participants in all experiments exceeded the recommended sample size suggested by the large effect size (at least Cohen's $d=0.70$ ) found with RB and by a power analysis using an alpha level of .05 and the G*Power software (Faul, Erdfelder, Lang, \& Buchner, 2007). The participants in this and the remaining experiments described herein had normal or corrected-to-normal vision, as well as English as a first language, and received partial course credit.

Stimulus materials and design The stimulus materials included all capital letters except the visually similar letters I and $\mathrm{L}$ and $\mathrm{U}$ and $\mathrm{V}$. The 100 RSVP trials were composed of 20 trials that included four unrepeated letters - that is, one presentation of the target letter (e.g., A-B-C-D); 20 trials that included one repetition - that is, two presentations of the target letter with one intervening item between the repeated items (e.g., A-B-A-D); 20 trials that included a group-that is, three uninterrupted presentations of the target letter (e.g., AA-A-D); 20 trials composed of only three unrepeated letters (e.g., A-B-C); and 20 trials composed of only two unrepeated letters (e.g., A-B). The letters for the trials were chosen randomly, with the exception that care was taken to ensure that the sequence did not spell an English word. Analyses were performed only on the three conditions that contained four items (unrepeated, repeated, and grouped trials). Trials with less than four letters were intended to reduce guessing on the basis of the knowledge that all trials should contain four items, and they were not included in the analyses.
Presentation order of trials was randomized. Trials were preceded and followed by stimulus masks composed of a row of four asterisks, the duration of which was determined on an individual basis, as described below. Turquoise letters were presented centrally and sequentially in size 14 Times New Roman font on a black background.

The independent variable was the number of target letter presentations (one, two, or three), and the dependent variables included both the mean accurate RT (time taken by participant to respond when accurate) and the mean percentage correct for each level of the independent variable (how often each participant correctly indicated the number of target items).

Apparatus and procedure Participants performed this task individually in normal room illumination. The task was executed on a PC using the Windows XP operating system and the DirectRT software (Jarvis, 2010). Responses were made on a DirectRT-compatible button bar labeled for the number of target items seen (zero through four), along with a button designated to initiate each trial. Each buttonpress was mapped to corresponding numbers in the output file.

Determination of exposure duration The amount of time that the stimuli were presented (exposure duration) was determined on an individual basis due to individual variation in the speed required for RB. Each participant viewed approximately 15 trials composed of four sequentially presented, unrepeated letters. After viewing the string, they reported the letters to the experimenter. The exposure duration was initially set at $156 \mathrm{~ms}$ per letter and decreased by $14 \mathrm{~ms}$ until the participant's accuracy was reduced to approximately $50 \%$. ${ }^{1}$ The presentation rate at which this was achieved for a given individual was then used for the experimental trials. The modal exposure duration for this experiment was $58 \mathrm{~ms}$, with a range of 58-114 ms. This initial setting was sufficient for the duration of the experiment, due to a lack of practice effects observed with RB (Kanwisher et al., 1996).

Experimental trials After the exposure duration trials, participants viewed the following instructions on the computer screen:

In this task, you will see a rapidly presented list of letters. There may be as few as two or as many four letters in each trial. At the start of each trial a single letter will be presented, this is the target letter. After all the letters have been presented, you will be prompted to indicate how many times you saw the target letter. As soon as

\footnotetext{
${ }^{1}$ On the basis of a pilot study, this cutoff was found to translate to the desired accuracy rate of $75 \%$ for unrepeated items in the experimental task.
} 
you have a single numerical response in mind, respond with the corresponding number on the keypad.

Participants then performed five practice trials of the experimental task in order to familiarize them with the task demands. First, participants pressed a button labeled "OK" to begin the trial. Then they viewed a single letter for 1 , $000 \mathrm{~ms}$, followed by a blank screen for $500 \mathrm{~ms}$. Then they viewed a mask composed of a row of four asterisks that remained on screen for a length of time corresponding to their predetermined exposure duration. Following the mask, they viewed a series of sequentially presented letters, with each letter displayed for the same predetermined exposure duration. A postmask of a row of four asterisks was presented for the same duration. After the mask, participants saw the prompt "How many?" which remained on the screen until the participant pressed a button that corresponded to the number of times they had seen the target letter. The experimenter remained in the room during the practice trials to ensure that the participants understood the task. If they did not understand, the experimenter repeated the instructions aloud.

After the practice trials, the experimenter left the room. The trials continued in the same way for the above-described 100 trials.

\section{Results}

Separate one-way, repeated measures analyses of variances (ANOVAs) were conducted on the accuracy (mean percentages correct) and RT (means for correct responses) data. Pairwise comparisons were made with Bonferroni corrections. Cases were removed if a participant's accuracy was below $35 \%$ overall, and individual trials were removed if they exceeded 2.5 standard deviations of the condition's mean score for RTs. All following analyses were conducted in this manner unless otherwise stated.

The ANOVA for accuracy revealed a large main effect of number of presentations, $F(2,36)=27.20, p<.001, \eta^{2}=.60$. As compared to the unrepeated condition $(M=71 \%, S D=$ $18 \%, 95 \%$ confidence interval $[\mathrm{CI}]=62 \%-79 \%$ ), we observed RB effects at both two $(M=42 \%, S D=22 \%$, $\mathrm{CI}=32 \%-53 \%, p=.001)$ and three $(M=40 \%, S D=$ $28 \%, \mathrm{CI}=27 \%-52 \%, p=.002)$ presentations. The results are presented graphically in Fig. 1.

Twenty-four trials were removed from the RT data (nine trials from the unrepeated condition, four trials from the repeated condition, and 11 trials from the grouped condition), or a total of $2 \%$ of the data. Analysis of the RTs revealed a large main effect of number of item presentations, $F(2,36)=6.60$, $p=.004, \eta^{2}=.27$. A possible item enumeration strategy was observed, since three presentations $(M=1,311 \mathrm{~ms}, S D=$ $327 \mathrm{~ms}, \mathrm{CI}=1,154-1,469 \mathrm{~ms})$ of the critical item elicited longer RTs than both one $(M=1,066 \mathrm{~ms}, S D=300 \mathrm{~ms}$, $\mathrm{CI}=927-1,216 \mathrm{~ms}, p=.028)$ and two $(M=1,148 \mathrm{~ms}, S D=$ $301, \mathrm{CI}=1,004-1,294 \mathrm{~ms}, p=.008)$ presentations. Although no other statistical differences were observed, the RT differences across conditions are at least numerically consistent with an item enumeration strategy. Descriptive statistics are presented in Fig. 2.

\section{Discussion}

RB effects were observed both when a single repetition was present and when three identical letters were presented consecutively in an RSVP display. It also appears that participants approached this task using an item enumeration strategy, whereby each instance of the target letter was counted. Supporting evidence for this claim was not as strong as expected, however, because a statistical difference was found only between one and three presentations of a letter. Interestingly, the accuracy rates in the RB and grouped conditions were nearly identical $(42 \%$ and $40 \%$, respectively). Given that grouped presentation provides more opportunities for RB to occur (in the form of more items for $\mathrm{RB}$ to act on), one would expect that a larger error rate should occur in that condition. This expectation is based on probability, rather than the prior literature, and no explanation of this facet of the results is considered at present.

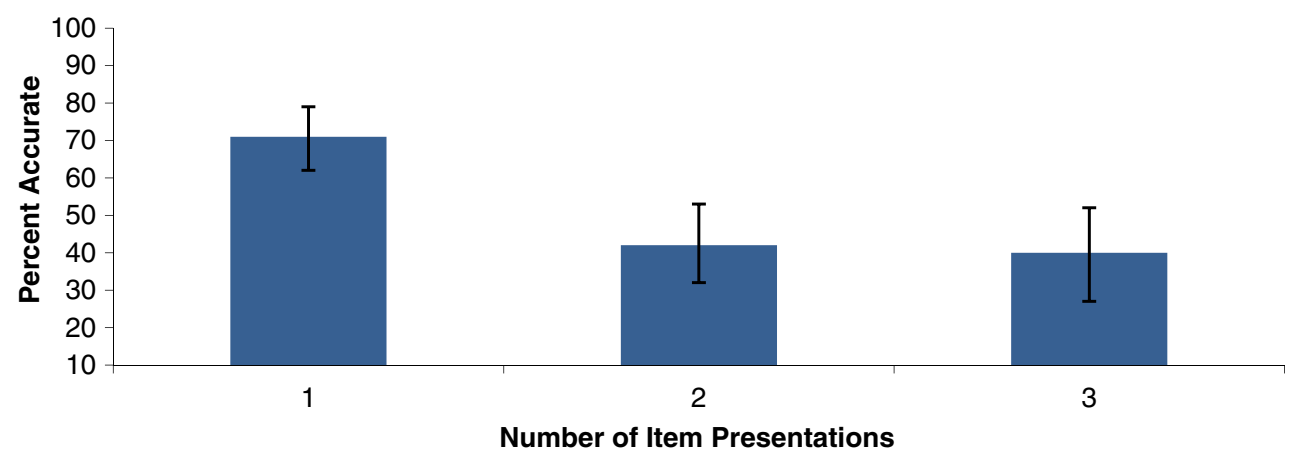

Fig. 1 Mean accuracy by condition. Error bars represent $95 \%$ confidence intervals 


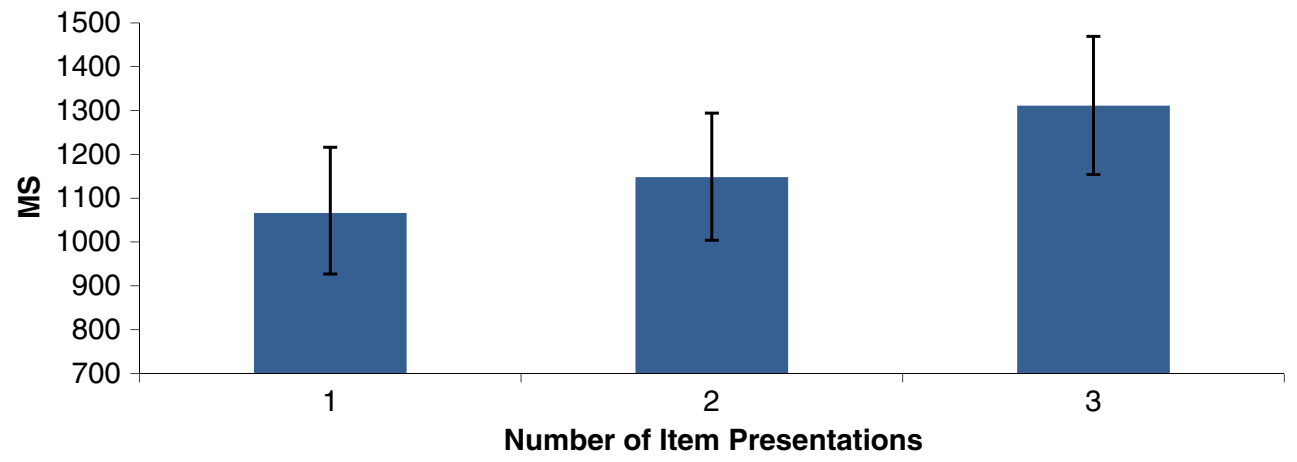

Fig. 2 Mean reaction times by condition. Error bars represent $95 \%$ confidence intervals

\section{Experiment 2}

Experiment 2 was designed to be a BSVP complement to Experiment 1, whereby letters were presented simultaneously as opposed to sequentially.

\section{Method}

Participants Thirty-three University of Windsor undergraduate psychology students (30 female, three male; mean age $=$ 20.18 years, age range $=18-27)$ participated in this experiment.

Stimulus materials and design The stimulus materials and design were the same as in Experiment 1, with the exception that letters were presented using a BSVP format. The display as a whole was centrally located on the computer monitor, with items presented simultaneously in the four quadrants of a square that was contained within $4 \times 4$ deg of visual angle. The duration of that presentation was individually determined, as we describe below. Each letter was presented within 2 deg of visual angle from the center of the square. This layout was chosen because it was thought that a traditional single-line presentation might bias viewers toward a left-to-right reading strategy.

Apparatus and procedure The apparatus and procedure were similar to those used in Experiment 1.

Determination of exposure duration Exposure duration was again determined individually. Participants viewed approximately 15 trials, composed of four simultaneously presented, unrepeated letters in the above-described square shape. The exposure duration was initially set at $170 \mathrm{~ms}$ and decreased by $14 \mathrm{~ms}$ until the participant's accuracy was reduced to approximately $50 \%$. The presentation rate at which this was achieved was then used for the experimental trials. This procedure resulted in a modal exposure duration of $72 \mathrm{~ms}$, with a range of 58-156 ms.

\section{Results}

The analysis procedures mimicked those of Experiment 1 . The ANOVA for accuracy revealed a large main effect of number of presentations, $F(2,64)=28.40, p<.001, \eta^{2}=$ 47. Relative to the unrepeated condition $(M=86 \%, S D=$ $10 \%, \mathrm{CI}=82 \%-90 \%)$, RB effects were found at two $(M=$ $71 \%, S D=22 \%, \mathrm{CI}=64 \%-80 \%, p<.001)$ and three $(M=$ $54 \%, S D=31 \%, \mathrm{CI}=43 \%-65 \%, p<.001)$ presentations of the target. More errors were also made in the grouped condition than in the two-presentation condition $(p=.002)$. Descriptive statistics are presented in Fig. 3.

Forty-seven outlier trials were removed from the RT data, a total of $2 \%$ of the data. Whereas the ANOVA for RTs revealed a main effect of number of presentations, $F(1.42,43.88)=$ $3.64,{ }^{2} p=.049, \eta^{2}=.11$, no differences were found among the pairwise comparisons.

This lack of differences may be due to statistical power being reduced as a result of participants adopting different strategies, which would increase the variability of the results. Support for this possibility comes from the observation of a group of individuals who could be classified as "high-accuracy performers" in the grouped condition (three presentations), despite overall reductions in accuracy relative to Experiment 1. To test this possibility, participants were assigned post-hoc to groups on the basis of their accuracy on the trials that contained three presentations of the target. The mean accuracy of the high-accuracy group was $80 \%(n=16)$, and the mean accuracy of the low-accuracy group was $30 \%(n=17)$. An independent-samples $t$ test compared the mean RTs of the two groups. The low-accuracy performers were thought to be using an item enumeration strategy and were expected to have increased RTs over the high-accuracy performers, who were assumed to be using a faster, familiarity-based strategy. The groups did differ, $t(30)=2.40, p=.023, \eta^{2}=.40$, in the hypothesized direction, with high-accuracy performers

\footnotetext{
${ }^{2}$ Here and later, Greenhouse-Geisser corrections have been applied where appropriate for violation of the assumption of homogeneity of variances.
} 


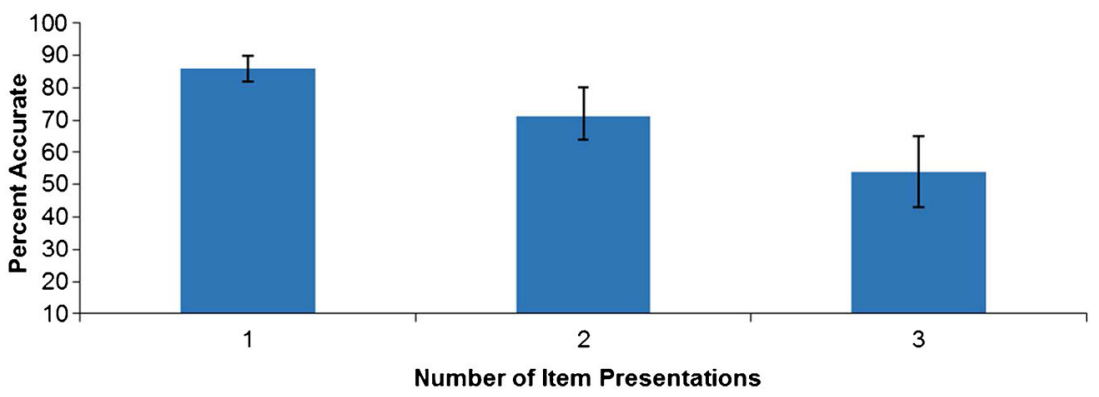

Fig. 3 Mean accuracy by condition. Error bars represent $95 \%$ confidence intervals

producing faster RTs $(M=1,003 \mathrm{~ms}, S D=150 \mathrm{~ms}, \mathrm{CI}=923$ $1,082 \mathrm{~ms})$ than the low-accuracy performers $(M=1,230 \mathrm{~ms}$, $S D=348 \mathrm{~ms}, \mathrm{CI}=1,045-1,416 \mathrm{~ms})$. See Fig. 4 for the data displayed graphically.

\section{Discussion}

The results of this experiment indicate that although BSVP presentation allows perceptual grouping to occur, it does not guarantee it. In fact, the data suggested that there might be groups of high- and low-accuracy performers, with the lowaccuracy performers producing relatively slow RTs indicative of an item enumeration strategy, whereas the high-accuracy performers produced faster RTs, consistent with a familiaritybased strategy, or an perhaps a modified item enumeration strategy whereby the grouped presentation was perceived as a single item. These results support the notion that linguistic stimuli can be grouped in a somewhat nontraditional way (i.e., into groups of three identical letters rather than into groups that form words) and that this grouping may depend on an individual's strategy choice.

\section{Experiment 3}

In Experiment 3, we evaluated whether grouping or RB would be observed for words presented in RSVP as opposed to letters and whether different strategies would be used.

\section{Method}

Participants Twenty University of Windsor undergraduate psychology students (18 female, two male; mean age = 20.75 years, age range $=18-34$ ) participated in this experiment.

Stimulus materials and design The stimulus materials consisted of four-letter words with an orthographic neighborhood between three and four (Durda \& Buchanan, 2006). Words with such low orthographic neighborhoods have been shown to be recalled better in RB tasks and should reduce the likelihood of orthographic similarity between unrepeated items (Coltheart \& Langdon, 2003; Morris \& Still, 2008). Although the words varied by orthographic frequency, the average frequency of each condition did not (see Appendix A). As with the letter trials in Experiments 1 and 2, the words for each trial were selected randomly from the list. Words containing the letter $\mathrm{M}$ or $\mathrm{W}$ were excluded so that the same stimuli could be used in the BSVP version in Experiment 4, in which the width of these letters would have prevented the item from properly fitting within 2 deg of visual angle from the display's center. In all other respects, this task was the same as that in Experiment 1, with trials consisting of either one, two, or three presentations of the target word.

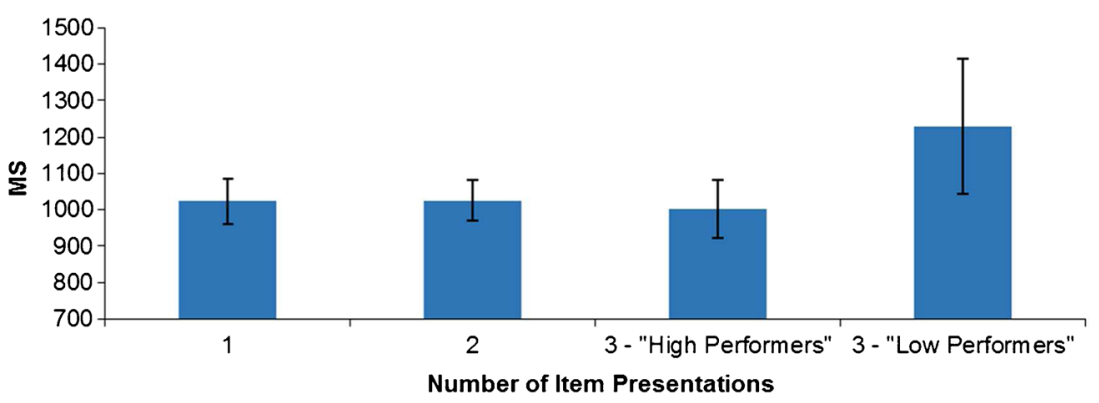

Fig. 4 Mean reaction times by condition, separated at three presentations by "high" and "low" performers. Error bars represent $95 \%$ confidence intervals 


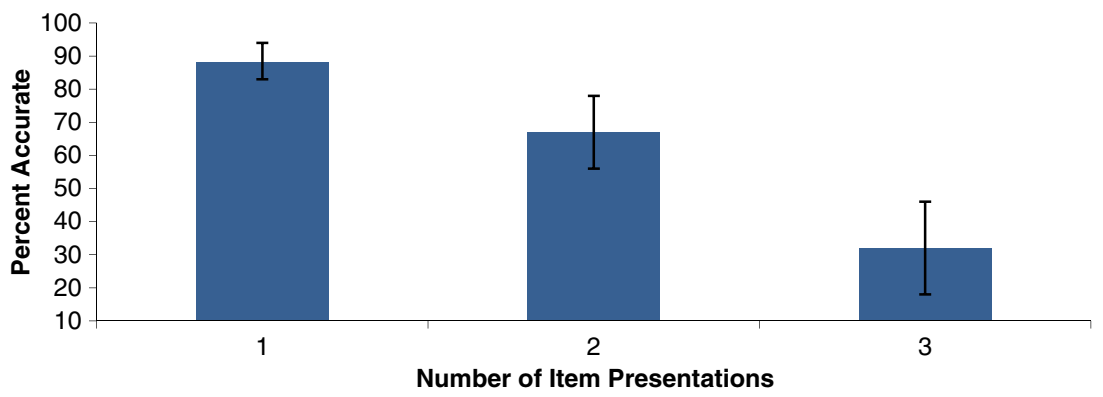

Fig. 5 Mean accuracy by condition. Error bars represent $95 \%$ confidence intervals

Apparatus and procedure The apparatus and procedure were the same as in Experiment 1, with the exception that words instead of letters were used as the items. The modal exposure duration for this experiment was $100 \mathrm{~ms}$, and the range was 86-142 ms.

\section{Results}

The ANOVA for accuracy revealed a large main effect of number of presentations, $F(1.50,28.56)=27.23, p<.001$, $\eta^{2}=.59$ (see note 2 ). As compared to the unrepeated condition $(M=88 \%, S D=12 \%, \mathrm{CI}=83 \%-94 \%)$, an RB effect was found at two $(M=67 \%, S D=24 \%, \mathrm{CI}=56 \%-78 \%, p=$ $.002)$ and three $(M=32 \%, S D=30 \%, \mathrm{CI}=18 \%-46 \%, p<$ $.001)$ target presentations. The accuracy at three presentations was lower than the accuracy at two presentations $(p=.003)$. Descriptive statistics are presented in Fig. 5.

Sixteen outlier cases were removed from the RT analysis, a total of $2 \%$ of the data. The ANOVA for RTs showed a large effect of number of presentations, $F(1.34,16.11)=11.31, p=$ $.002, \eta^{2}=.49$. A possible item enumeration strategy was found, since three presentations of the critical item $(M=1$, $202 \mathrm{~ms}, S D=231 \mathrm{~ms}, \mathrm{CI}=1,068-1,335 \mathrm{~ms}$ ) elicited a longer RT than did one presentation $(M=946 \mathrm{~ms}, S D=183 \mathrm{~ms}, \mathrm{CI}=$ 841-1,052 ms, $p=.002)$. The mean differences between one and two presentations $(p=.08)$ and two and three presentations $(p=.095)$ were in the direction anticipated by an item enumeration strategy, but only approached significance. Descriptive statistics are presented in Fig. 6.

\section{Discussion}

The results of Experiment 3 mirror those of Experiment 1: RB was observed at two and three presentations of a word, and the pattern of RTs across conditions was indicative of an item enumeration strategy.

\section{Experiment 4}

Using the same set of words, in Experiment 4 we sought to determine the effects of presenting these items in a BSVP format.

\section{Method}

Participants The participants were 20 University of Windsor undergraduate psychology students (18 female, two male; mean age $=19.75$ years, age range $=18-34$ ).

Stimulus materials and design The stimulus materials and design were the same as in Experiment 3, with the exception that items were presented using the same BSVP format described in Experiment 2.

Apparatus and procedure The apparatus and procedure were the same as we had used in previous experiments, with the exception that the modal exposure duration for the items was $114 \mathrm{~ms}$, with a range of 72-184 ms.

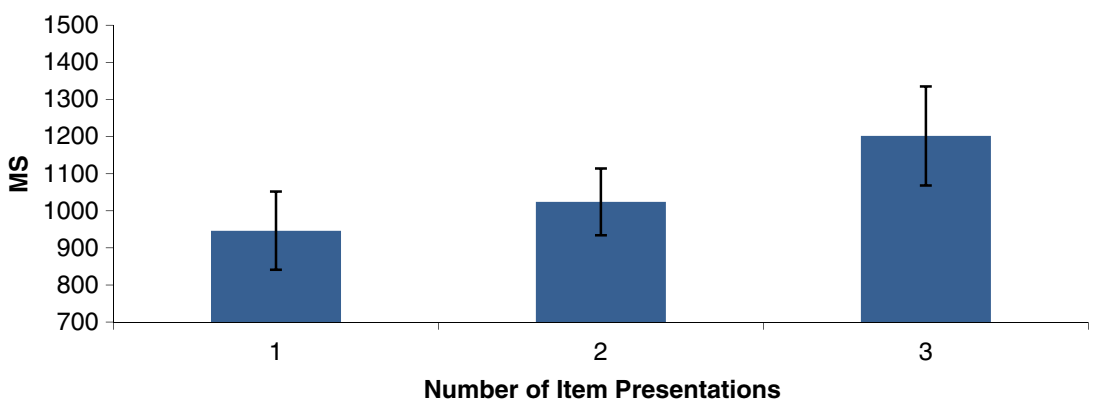

Fig. 6 Mean reaction times by condition. Error bars represent $95 \%$ confidence intervals 


\section{Results}

One participant in this experiment was excluded due to belowcutoff performance. The ANOVA for accuracy found a large main effect of number of presentations, $F(2,36)=27.20, p<$ $.001, \eta^{2}=.60$. As compared to the unrepeated condition $(M=$ $74 \%, S D=18 \%, \mathrm{CI}=66 \%-83 \%), \mathrm{RB}$ effects were found at two $(M=45 \%, S D=25 \%, \mathrm{CI}=33 \%-57 \%, p<.001)$ and three $(M=41 \%, S D=26 \%, \mathrm{CI}=28 \%-54 \%, p<.001)$ presentations. No differences were observed between two and three presentations. Descriptive statistics are presented in Fig. 7.

Nineteen individual trials were removed from the RT analysis, a total of $3 \%$ of the data. The ANOVA for mean accurate RTs did not reveal an effect of number of presentations, but the analysis was underpowered $F(1.45,26.09)=1.96 p=.155, \eta^{2}=.10$, observed power $=.38$ (see note 2). As in Experiment 2, we suspected that a mix of strategies was complicating the picture. A split of high-accuracy performers $(M=69 \%$, $n=7)$ from low-accuracy performers $(M=25 \%, n=$ 12) was made, followed by an independent-samples $t$ test of their mean accurate RTs.

We again hypothesized that the low-accuracy performers were likely using an item enumeration strategy, and thus would have slower RTs. The high-accuracy performers, on the other hand, were suspected to be achieving their success by using a familiarity-based strategy or by viewing the group as a single item, and would likely display shorter RTs. The two groups did differ, $t(17)=2.504, p=.023$, and the highaccuracy performers $(M=950 \mathrm{~ms}, S D=158 \mathrm{~ms}, \mathrm{CI}=804$ $1,097 \mathrm{~ms}$ ) did have reliably faster RTs than the low-accuracy performers $(M=1,137 \mathrm{~ms}, S D=157 \mathrm{~ms}, \mathrm{CI}=1,038-1$, $237 \mathrm{~ms}$ ), and thus the two groups were likely using different strategies. See Fig. 8 for a graphic display.

\section{Discussion}

As with the previous experiments, the data overall indicated $\mathrm{RB}$ at two and three presentations of a word. Overall, the pattern of RTs appeared flat across conditions, suggesting either a familiarity-based strategy or that the grouped presentation was perceived as a single item. However, when our procedure of comparing high- and low-accuracy performers was used, the RT patterns also diverged in a predictable manner. As in Experiment 2, the participants exhibiting greater accuracy also had faster RTs than the participants exhibiting poorer accuracy.

\section{Experiment 5}

Experiment 5 was designed to address limitations of the previous four experiments. Specifically, in the earlier experiments discovery and statistical analysis of the groups were performed post-hoc, and therefore were largely exploratory. A lack of randomization across experiments also prohibited cross-experiment comparisons. Additionally, an explicit question about strategy, which would have provided converging support, was not asked. Finally, the procedures to determine the exposure duration did not consistently yield comparable accuracy rates in their corresponding experiments (i.e., accuracy was generally higher in the formal experiments than in the procedure designed to determine the exposure duration). Given that the high-accuracy performers in multiple experiments were found to have higher accuracy across all target presentations, the possibility existed that the results of this group were largely driven by individuals who were performing at ceiling.

Accordingly, in Experiment 5 we directly tested the existence of groups of high- and low-accuracy performers, randomized participants across experiments, asked an explicit question regarding strategy, and addressed ceiling-level performance by reducing the exposure duration across participants. It was hypothesized that (1) groups of high- and lowaccuracy performers would be found; (2) the high-accuracy performers would have faster, flatter RTs across target presentations than the low-accuracy performers; and (3) high- and low-accuracy performers could be differentiated on the basis of strategy use, with high-accuracy performers using nonsequential strategies (perceiving the display as a whole) and low-accuracy performers using sequential strategies (rapid reading).

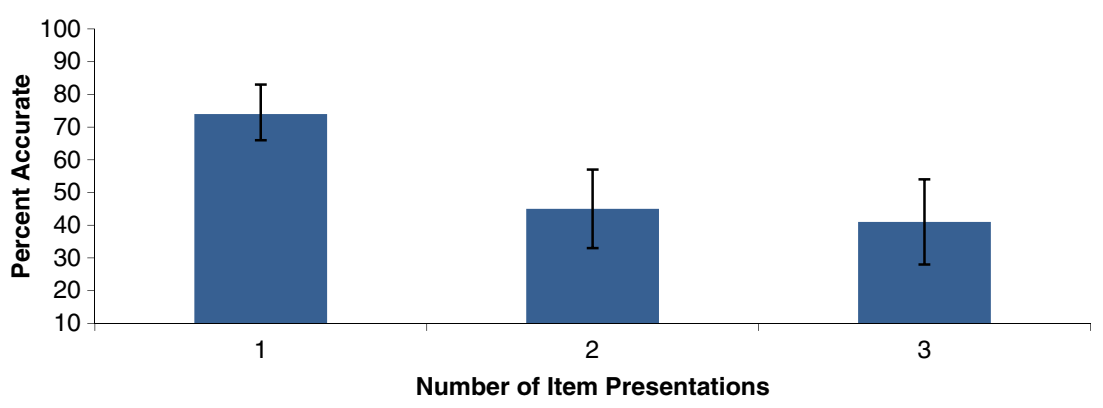

Fig. 7 Mean accuracy by condition. Error bars represent $95 \%$ confidence intervals 


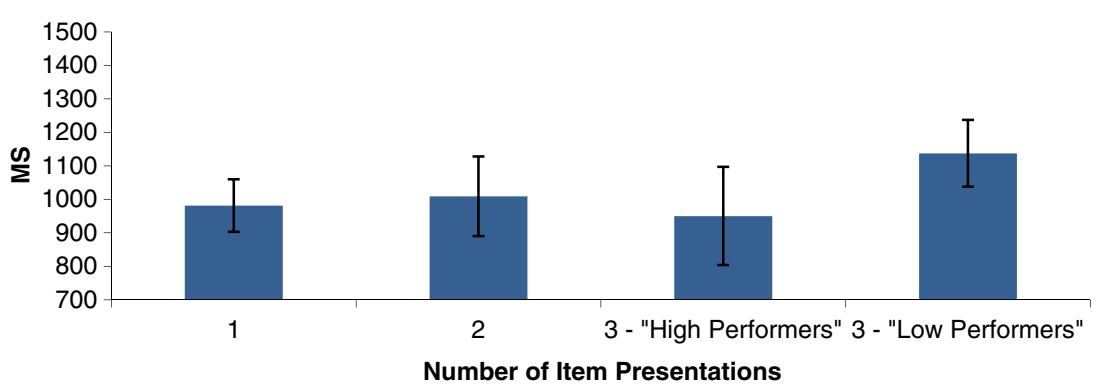

Fig. 8 Mean reaction times by condition, separated at three presentations by "high" and "low" performers. Error bars represent $95 \%$ confidence intervals

\section{Method}

Participants The participants were 80 University of Windsor undergraduate psychology students (70 female, ten male; mean age $=21$ years, age range $=18-35$ )

Stimulus materials and design The stimulus materials included the four above-described experiments, compiled into a $2 \times 2 \times 3$ mixed design with one between-subjects variable (format) with two levels (RSVP or BSVP) and two withinsubjects variables. The within-subjects variables included stimuli (words or letters) and targets (one, two, or three presentations). Participants were randomized to either the RSVP or BSVP condition and viewed either letters before words or words before letters in a randomized, counterbalanced design. The exposure duration was set for all participants by determining the modal value for each condition (format and stimuli) from previous experiments and subtracting $14 \mathrm{~ms}$. For example, the modal exposure duration for Experiment 4 was $114 \mathrm{~ms}$. Using the above-described formula, the exposure duration for the BSVP words condition in the present experiment was $100 \mathrm{~ms}$. For one condition, RSVP letters, the calculated exposure duration yielded unacceptably low (below the cutoff of $35 \%$ ) accuracy in pilot testing. Therefore, the modal value from Experiment 1, rather than the modal value minus $14 \mathrm{~ms}$, was used.

Apparatus and procedure The apparatus and procedure were the same as in the previous experiments, with the exception that the modal exposure duration for each condition was preset (RSVP letters, $58 \mathrm{~ms}$; BSVP letters, $58 \mathrm{~ms}$; RSVP words, $86 \mathrm{~ms}$; BSVP words, $100 \mathrm{~ms}$ ), the participants each performed two tasks (RSVP letters and words or BSVP letters and words), and the participants answered a multiple-choice question at the end of each condition asking explicitly about their primary strategy. The options were drawn from pilot data in which participants were asked an open-ended question about strategy, and included (1) I read each item as quickly as possible, (2) I viewed the items as part of a larger whole, (3) I went with my gut, (4) I mostly guessed, and (5) other.

\section{Results}

Separate mixed between-within-subjects ANOVAs were conducted on the accuracy (mean percentages correct) and RT data at each level of the independent variable (number of targets) in order to elucidate overall trends in the data and to determine whether the data could be collapsed across conditions in subsequent analyses. Five participants in this experiment performed below the accuracy cutoff, and therefore their data were not included in the analyses. Four of these participants had completed the RSVP version of the task, with the other performing the BSVP version.

The overall ANOVA for accuracy by participants revealed main effects for number of targets, $F(1.95,138.51)=132.83{ }^{3}$ $p<.001, \eta^{2}=.65$, and format, $F(1,71)=26.34, p<.001, \eta^{2}=$ .27 . No main effects of stimuli or order were found, but multiple interactions were observed involving both stimuli and order. Accordingly, the types of stimuli were analyzed separately with condition as a between-subjects variable, and order was entered as a covariate in subsequent analyses.

Assignments to high- and low-accuracy groups were largely based on the pattern of performance observed in previous experiments. Specifically, inclusion in the high-accuracy group was based on meeting one of two criteria: (1) accuracy at three presentations greater than accuracy at two presentations or (2) accuracy approximately equivalent across conditions (accuracy at one and three presentations within $5 \%$ ). These criteria were meant to capture those who did not exhibit $\mathrm{RB}$ at three presentations but might or might not have exhibited RB at two presentations. Accordingly, 21 participants were classified as high-accuracy performers when the stimuli were letters (54 low-accuracy performers), and 25 participants were classified as high-accuracy performers when the stimuli were words (50 low-accuracy performers). Interestingly, group membership was not entirely consistent between the stimuli. Specifically, whereas the correlation between group membership for letters and words was significant, $r=.315, p=$ .006 , only 53 of 75 participants remained in the same group across stimuli.

\footnotetext{
${ }^{3}$ The Huynh-Feldt correction was applied for violation of the assumption of homogeneity of variances.
} 
The ANOVA on accuracy for letters by group membership and format yielded main effects of group membership, $F(1$, $70)=27.34, p<.001, \eta^{2}=.28$, with those classified as highaccuracy performers unsurprisingly yielding higher accuracy overall [by format, $F(1,70)=37.56, p<.001, \eta^{2}=.35$, with overall higher accuracy in BSVP conditions; by targets, $F(2$, $140)=7.23, p=.001, \eta^{2}=.09$, with accuracy decreasing as the number of targets increased]. A group membership by targets interaction was found, $F(2,140)=52.59, p<.001$, $\eta^{2}=.43$. Follow-up independent-samples $t$ tests revealed that those in the high-accuracy group were found to have higher accuracy only at two, $t(73)=2.09, p=.040$, and three, $t(73)=$ $9.77, p<.001$, target presentations. A format by targets interaction was also found, $F(2,140)=4.15, p=.018, \eta^{2}=.056$, suggesting a more linear, but shallow, decline in accuracy as targets increased in the BSVP condition (one target $>$ two targets $>$ three targets) than in the RSVP condition, which evidenced a sharp decline in accuracy from one to two target presentations, but no additional decline in accuracy from two to three target presentations. Descriptive statistics are presented in Fig. 9.

With regard to strategy use for letters, the most commonly used strategy was perceiving letters as shapes $(n=32)$, followed by rapid reading $(n=19)$, and perceiving the display as a whole $(n=17)$. The use of rapid reading versus perceiving the display as a whole was not found to be associated with group membership, $p=.59$. Those who viewed the displays as a
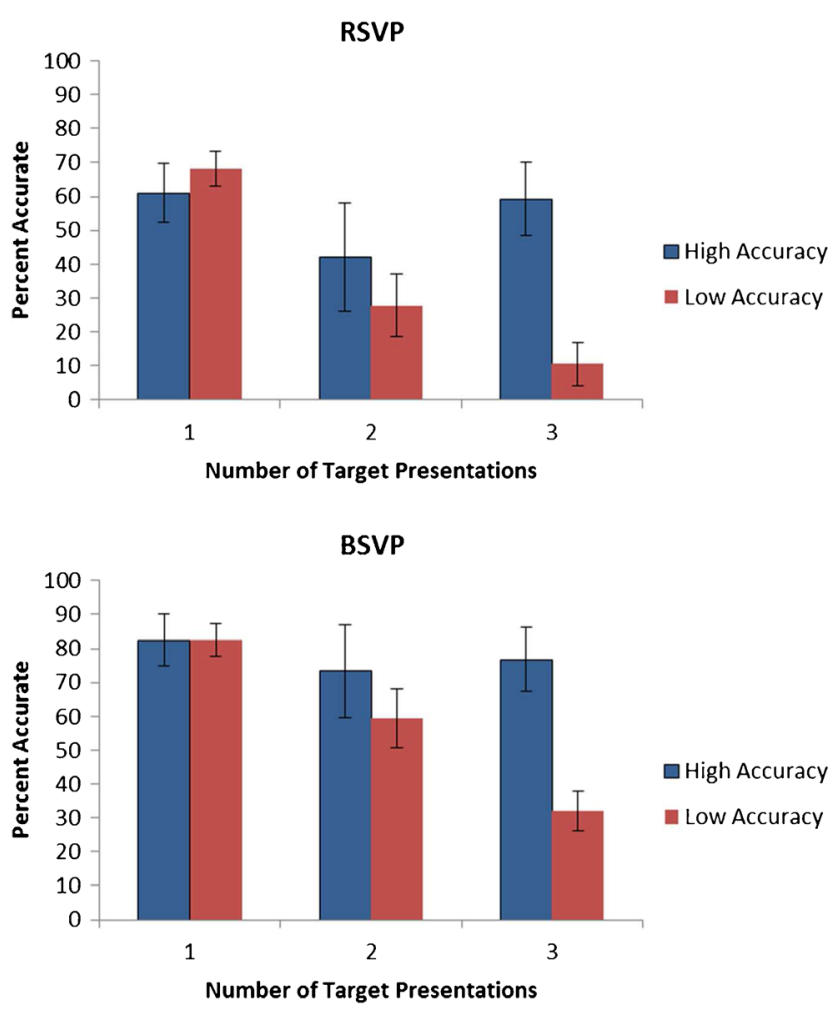

Fig. 9 Mean accuracy for letters by format, group, and targets. Error bars represent $95 \%$ confidence intervals whole were likewise not found to have higher accuracy at three presentations of the target $(M=47.65 \%)$ than those who read rapidly $(M=31.05 \%), t(34)=1.72, p=.095$.

The ANOVA on accuracy for words by group membership yielded results nearly identical to those from the analysis of letters. Again, main effects were found for group, $F(1,70)=$ $12.51, p=.001, \eta^{2}=.15$, with those classified as highaccuracy performers again yielding higher accuracy overall [by format, $F(1,70)=6.60, p=.012, \eta^{2}=.09$, with greater accuracy for BSVP than for RSVP; by number of targets, $F(1.88,131.52)=7.47, p=.001, \eta^{2}=.10$ (see note 2$)$, whereby accuracy was greater at one target presentation than for two and three target presentations]. Identical interactions were also found. Specifically, a group membership by targets interaction emerged, $F(1.88,131.52)=24.31, p<.001, \eta^{2}=.26$, with follow-up $t$ tests revealing that high- and low-accuracy performers only differed in accuracy at three target presentations. A format by targets interaction was also observed, $F(1.88$, $131.52)=9.75, p<.001, \eta^{2}=.12$, with a greater drop in accuracy at two and three target presentations in the RSVP than in the BSVP condition. Descriptive statistics are presented in Fig. 10.

The strategy choices for words also mirrored those for letters. Specifically, the most frequently used strategies included rapid reading $(n=24)$, perceiving the display as a whole $(n=$
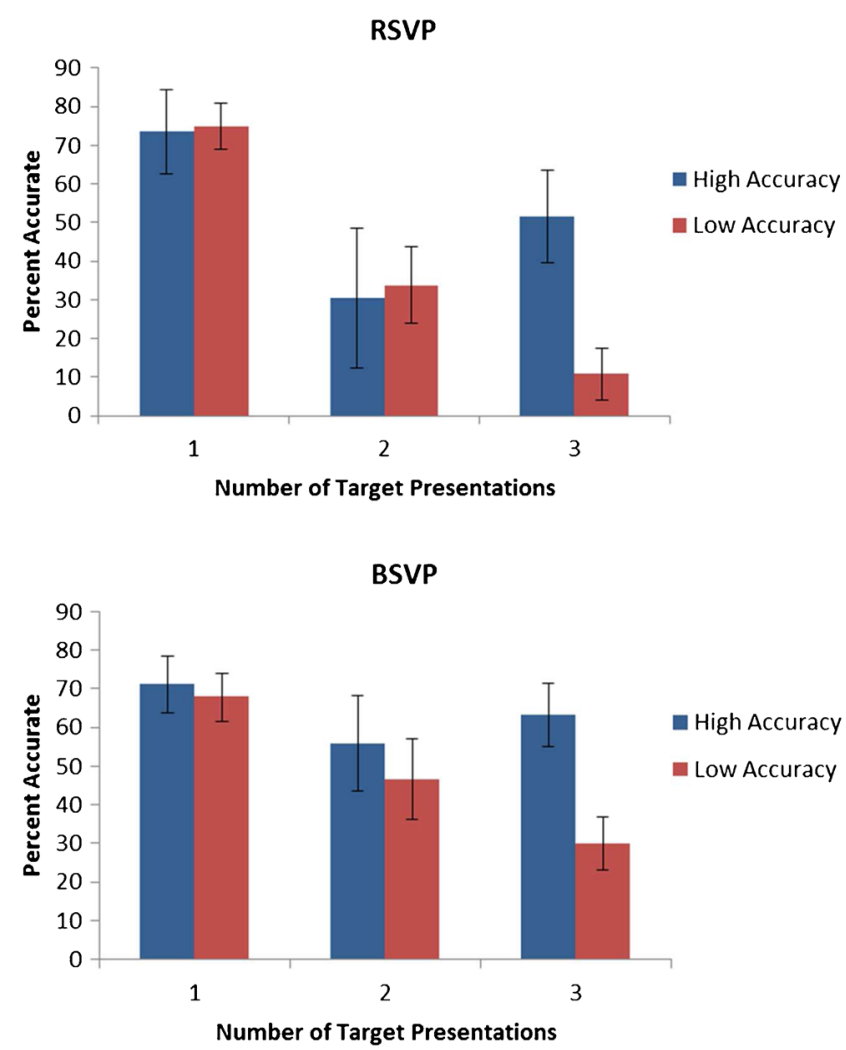

Fig. 10 Mean accuracy for words by format, group, and targets. Error bars represent $95 \%$ confidence intervals 
$25)$, and focusing only on the shapes of the words $(n=20)$. Group membership was again not associated with the use of rapid reading or perception of the whole, $p=.21$. However, use of the latter strategy did yield higher accuracy at three target presentations, as compared with rapid reading, $t(47)=$ $2.03, p=.05$.

Ninety-one outlier cases were removed from the RT analysis, a total of $1.8 \%$ of the data. One additional participant was also removed from the analysis after an analysis of outliers. The overall ANOVA for RTs showed main effects of format, $F(1,50)=9.62, p=.003, \eta^{2}=.16$, with BSVP being faster than RSVP; order, $F(1,50)=5.86, p=.019, \eta^{2}=.11$, with those who viewed letters first exhibiting faster RTs overall; stimuli, $F(1,50)=5.70, p=.02, \eta^{2}=.10$, with faster RTs for words than for letters; and number of targets, $F(1.54$, $77.15)=27.57, p<.001, \eta^{2}=.36$, with RTs increasing as the number of targets increased. Several interactions were also observed. Specifically, a stimuli by order interaction, $F(1,50)$ $=38.76, p<.001, \eta^{2}=.48$, revealed that RTs for letters were the same regardless of order, but that those who viewed letters first were faster when viewing words $(887.83 \mathrm{~ms})$ than were those who viewed words first $(1,141.58 \mathrm{~ms})$. A format by number of targets interaction was also observed, $F(1.54$, $77.15)=9.67, p=.001, \eta^{2}=.16$, with a steeper increase in RTs across targets emerging for RSVP than for BSVP. Descriptive statistics are presented in Fig. 11.

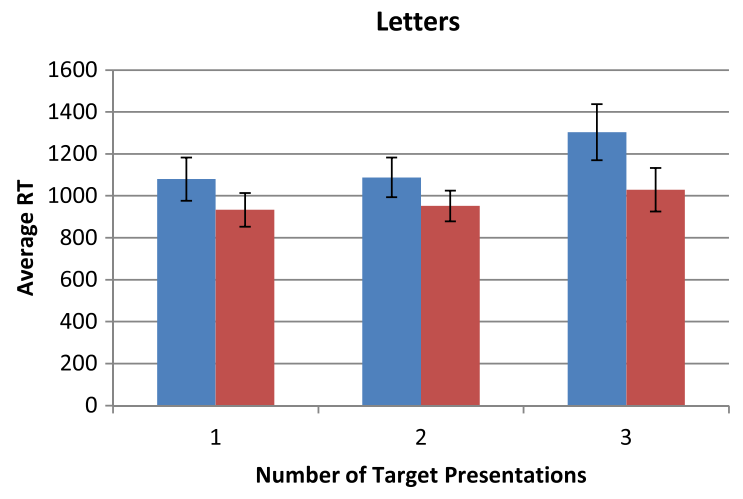

- RSVP - BSVP

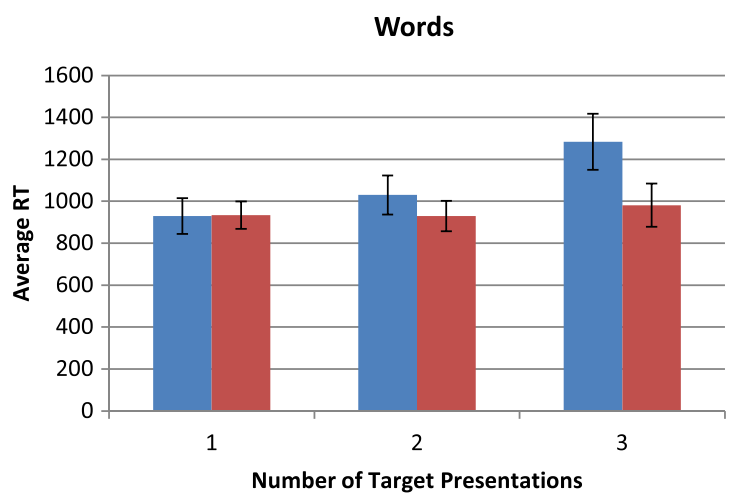

- RSVP BSVP

Fig. 11 Mean reaction times for letters and words by format, group, and targets. Error bars represent $95 \%$ confidence intervals
The ANOVA on RTs for letters by group membership (high-accuracy $n=21$, low-accuracy $n=36$ ) yielded no effect of group membership, $p=.46$. Likewise, the ANOVA on RTs for words by group membership (high accuracy $=24$, low accuracy $=36$ ) yielded no effect of group membership, $p=$ .291 .

\section{Discussion}

The results of Experiment 5 revealed that the two groups (high- and low-accuracy performers) were still distinguishable when overall accuracy was reduced via shortened exposure duration. Both high- and low-accuracy performers demonstrated equivalent accuracy at one target presentation, suggesting that the advantage shown by high-accuracy performers was not simply that they were better overall at detecting targets. Moreover, when just the word condition was examined, the groups only differed in accuracy at three target presentations, where "grouping" is hypothesized to occur.

With regard to strategy use as evidenced by RTs, the previous performance patterns were not found. Instead, both groups showed a uniform gradual increase in RTs with increased numbers of target presentations. This might be due to the fact that those with the fast, flat RTs were actually those who had performed at ceiling in previous experiments and who were necessarily eliminated in the present experiment.

Explicit questioning of strategy use also revealed no differences between the groups, with those viewing the displays as a whole being no more likely to be a member of the high- or low-accuracy performers. However, those who employed this strategy did tend to have higher accuracy at three target presentations overall. These findings suggest that explicit strategy may contribute to enhanced accuracy at three target presentations but is not a determining factor in group membership.

\section{General discussion}

Taken together, the data across these experiments reveal an overall pattern of repetition blindness across repeated and grouped conditions for letters and words displayed either sequentially or simultaneously. In other words, when linguistic stimuli are presented in traditional RB experimental conditions, the majority of responses to grouped items closely resemble Mozer's (1989) description of the homogeneity effect, with underestimation of repeated, identical items. ${ }^{4}$ This lack of grouping may reflect a reader's propensity to group letters into words and words into phrases, rather than to group

\footnotetext{
${ }^{4}$ The modal response to three target presentations was "two." The next most common response was "one," with few participants responding with "zero" or "four." The bigram frequency was not correlated with accuracy, suggesting no difference between common and uncommon bigrams.
} 
linguistic stimuli by identity. Such an assertion is further supported by Abrams and colleagues' (1996) finding that RB can be prevented by presenting phrases in syntactically appropriate groups, suggesting that participants perceived the phrase as a unit containing a group of words.

This pattern of decreasing accuracy as the number of target presentations increased appeared to be more pronounced in RSVP than in BSVP, with BSVP tasks often yielding a shallower decline in accuracy for both repeated and grouped trials. Reduced RB with BSVP has often been observed in the literature and has been explained by automatic coding of location, which helps to distinguish identical items, thus reducing RB (Epstein \& Kanwisher, 1999; Kanwisher, 1991; Luo \& Caramazza, 1996).

With regard to predominant patterns across the RT data, increasing RTs as the number of target presentations increased was consistently observed across tasks using an RSVP format. This result is consistent with a previous investigation into RB RTs by Wong and Chen (2009), who also found increased RTs as target presentations increased. Our RT data for BSVP tasks often yielded no differences based on the number of target presentations, but these analyses were underpowered. In the final experiment, BSVP RTs were found to increase as the number of target presentations increased, but as in the accuracy data, the slope of this increase was flatter than in tasks using RSVP. Additionally, participants responded more quickly to BSVP displays overall than to RSVP displays.

These RT patterns suggest that the majority of participants likely used an enumeration-based strategy, whereby instances of the target were mentally tallied before the response was given. This is further supported by the responses of those participants asked explicitly about strategy use. In terms of stated strategy use, the majority of participants noted either reading rapidly or scanning rapidly for the general shapes of the letters and words. In contrast, fewer participants reported viewing the display as a whole or using a "gut" response. When analyzed by format, however, the use of rapid reading or scanning was predominately found for RSVP as compared to the BSVP format. Specifically, BSVP tasks tended to yield more individuals who endorsed viewing the display as a whole and who exhibited either no differences in RTs by number of target presentations or a much shallower increase in RTs by number of target presentations.

Although the data overall demonstrate a general lack of grouping effects and an enumeration-based strategy, a compelling number of participants displayed a markedly different pattern of responding. Specifically, these participants generated a pattern of accuracy that more closely resembled that in Goldfarb and Treisman's (2011) results, with increased accuracy ("survival of the grouped") when viewing the grouped trials. This alternate pattern of performance first became evident in BSVP tasks, in which Goldfarb and Treisman hypothesized grouping would be most salient. Experiment 5 confirmed the existence of the two patterns of performance, while removing the possibility that those with increased accuracy for the grouped displays were just more accurate at detecting targets overall.

Although the RTs for this high-accuracy group were initially observed to be faster and flatter than those for their less accurate counterparts, suggesting a difference in strategy, reducing ceiling-level performance by decreasing exposure durations seemed to eliminate this difference. This suggestion of uniform strategy use may actually reflect strategy use at the time of recall, rather than encoding, as originally described by Brown and colleagues (2000). In other words, the two groups may initially perceive the stimuli differently, but use the same strategy to recall this perception when responding. However, explicit strategy uses also did not differ between groups, despite the fact that those who viewed the displays as a whole did have increased accuracy as compared to those who simply read the displays as quickly as they could.

Given this result, the individual differences in patterns of performance are more likely to be due to individual differences in responsiveness to the saliency of the grouped configuration than to explicit selection of a specific strategy or conscious effort to group items. In fact, the use of overt report has been found to be insufficient in measuring whether perceptual grouping has taken place (Lamy, Segal, \& Ruderman, 2006). Interestingly, individual differences in responsiveness to perceptual grouping have been found to vary systematically with reading ability in children. Specifically, the effect of grouping has been demonstrated to increase as reading ability decreases (Williams \& Bologna, 1985). Williams and Bologna suggested that this effect is due to poor readers being less proficient at selectively attending to items within a unit or group than are good readers. Accordingly, it is possible that highaccuracy performers may experience stronger grouping effects overall, as well as demonstrate difficulty attending to individual items within a group.

Kahneman and Treisman (1992) suggested that the creation of a token could be driven either by bottom-up (i.e., stimulus) factors, as was likely the case in these experiences, or by the allocation of attention. Similarly, it has been repeatedly demonstrated that the unit affected by RB is the unit that is attended (Epstein \& Kanwisher, 1999; Kanwisher, Driver, \& Machado, 1995; Kanwisher \& Potter, 1990). As such, it would be expected that those viewing the display as a whole would have more strongly demonstrated preserved perception for the grouped displays. The fact that this was not strongly demonstrated suggests that, despite viewing the display as a whole, participants still processed the items as individual units in order to comply with the task demands. In other words, using the example of a face as a multifaceted item, participants 
viewed the face, but still tallied up how many eyes were present, leaving them vulnerable to RB.

Alternatively, having fixed response options for the strategy question may have obscured important distinctions and nuances with regard to strategy. For example, participants were only allowed to select one response to represent their primary strategy when they may have used a combination of strategies or varied their strategies across the course of the task. Additionally, those who responded to a given response option may not actually represent a unified group, but distinct variations. This may be an explanation for why those who viewed the display as a whole performed better than those who did not at three target presentations, but viewing displays as a whole was not associated with being in the high-accuracy group.

The existence of the high- and low-accuracy groups also points to the main limitation of the study: Namely, it was assumed that use of a strategy would be consistent across individuals. Specifically, it was thought that the mere creation of groups (putting three items together in a display) would guarantee that participants would perceptually group the linguistic items if it were possible. However, physically grouping items does not appear to be sufficient, by itself, to produce grouping of linguistic stimuli among participants. Another important limitation was the relatively arbitrary criteria used to define the groups. Although a priori criteria for group membership were set, specifically the presence of a pattern similar to a "survival of the grouped" effect versus the presence of a pattern similar to a "homogeneity effect," this distinction was still largely based on experimenter discretion.

Further research will be needed in order to elucidate the conditions that lead participants to group linguistic stimuli as opposed to processing such stimuli sequentially. Specifically, systematic variation of bottom-up factors (e.g., proximity, color) and measurement of its effect on the number of participants who exhibit "survival of the grouped" would be of interest. Additionally, provision of explicit instructions to participants to group displays would be necessary to determine whether the allocation of attention and explicit strategy use can have a meaningful impact on accuracy. Finally, given the relationship between grouping and reading ability, exploration of reading ability and related factors (e.g., language dominance, bilingualism) and of the existence of a relationship with different processing strategies would be of benefit.

Repetition blindness is a robust effect, representing a failure at the interface of perception, semantics, and memory encoding. Investigation of RB therefore is able to inform all three of these cognitive domains. Specifically, it provides insight into how language is perceived, accessed, and stored in the brain. The results of this study, in particular, revealed that under traditional RB conditions, whether a "survival of the grouped" or a "homogeneity" effect is observed might depend on individual differences in sensitivity to the grouped items in a display.

Author note This research was supported in part by the Natural Sciences and Engineering Research Council of Canada.

\section{Appendix}

Table 1 Stimuli used in Experiments 3, 4, and 5

\begin{tabular}{|c|c|c|c|c|c|}
\hline \multicolumn{6}{|l|}{ Words } \\
\hline ABLE & DEFY & GOAL & LOAF & REEF & THUS \\
\hline ACHE & DENY & GOLF & NAVY & REIN & TIER \\
\hline ACID & DIAL & GORY & NEON & RISK & TOGA \\
\hline ACRE & DRIP & GREY & OAFS & ROSY & TROD \\
\hline ACTS & DROP & GULF & OAKS & RUIN & TROT \\
\hline AEON & DRUG & GULP & OILS & SALT & TUBA \\
\hline AIRY & DUAL & HALO & OILY & SELF & TUBE \\
\hline ALAS & DUCT & HOBO & ONTO & SHUN & TUNA \\
\hline ALOE & DULY & HURT & OPEN & SIGH & TURF \\
\hline ASKS & ERAS & HYPE & OURS & SIZE & TYKE \\
\hline AUNT & EVEN & ICES & OVER & SNIP & UNTO \\
\hline AVID & EXES & INKS & OXEN & SNUB & USER \\
\hline AXON & FISH & INTO & PITY & SOAK & VARY \\
\hline BIRD & FOLK & IRIS & PLAN & SODA & VEIN \\
\hline BLAB & FREE & IRKS & PLEA & SOFA & VERB \\
\hline BLIP & FROG & JOIN & PLUG & SOUL & VIAL \\
\hline BLOC & FUEL & JURY & POET & STUB & YAKS \\
\hline BODY & FUND & KEYS & PREP & STUD & YELP \\
\hline BRED & FURY & KNOB & PREY & STUN & YULE \\
\hline CHEF & FUSE & KNOT & PULP & SUCH & \\
\hline CHUG & GALA & LADY & PUTT & SURF & \\
\hline CLUE & GIRL & LAZY & QUAD & TECH & \\
\hline COAX & GLUT & LION & QUIZ & TEXT & \\
\hline DEBT & GNAT & LISP & RACY & THIS & \\
\hline
\end{tabular}

The stimuli were compiled from Wordmine2 (Durda \& Buchanan, 2006)

\section{References}

Abrams, L., Dyer, J. R., \& MacKay, D. G. (1996). Repetition blindness interacts with syntactic grouping in rapidly presented sentences. Psychological Science, 7, 100-104. doi:10.1111/j.1467-9280.1996. tb00337.x

Bavelier, D. (1994). Repetition blindness between visually different items: The case of pictures and words. Cognition, 51, 199-236. doi:10.1016/0010-0277(94)90054-X

Bavelier, D., \& Potter, M. C. (1992). Visual and phonological codes in repetition blindness. Journal of Experimental Psychology: Human 
Perception and Performance, 18, 134-147. doi:10.1037/00961523.18.1.134

Brown, N. R., Buchanan, L., \& Cabeza, R. (2000). Estimating the frequency of nonevents: The role of recollection failure in false recognition. Psychonomic Bulletin \& Review, 7, 684-691. doi:10.3758/ BF03213007

Coltheart, V., \& Langdon, R. (2003). Repetition blindness for words yet repetition advantage for nonwords. Journal of Experimental Psychology: Learning, Memory, and Cognition, 29, 171-185. doi: 10.1037/0278-7393.29.2.171

Coltheart, V., Mondy, S., \& Coltheart, M. (2005). Repetition blindness for novel objects. Visual Cognition, 12, 519-540. doi:10.1080/ 13506280444000427

Corballis, M. C., \& Armstrong, C. (2007). Repetition blindness is orientation blind. Memory \& Cognition, 35, 372-380. doi:10.3758/BF0. 193458

Durda, K., \& Buchanan, L. (2006). WordMine2 [Online]. Available at http://web2.uwindsor.ca/wordmine

Epstein, R., \& Kanwisher, N. (1999). Repetition blindness for locations: Evidence for automatic spatial coding in an RSVP task. Journal of Experimental Psychology: Human Perception and Performance, 25, 1855-1866. doi:10.1037/0096-1523.25.6.1855

Faul, F., Erdfelder, E., Lang, A.-G., \& Buchner, A. (2007). G*Power 3: A flexible statistical power analysis program for the social, behavioral, and biomedical sciences. Behavior Research Methods, 39, 175-191. doi:10.3758/BF03193146

Frick, R. W. (1987). The homogeneity effect in counting. Perception \& Psychophysics, 41, 8-16. doi:10.3758/BF03208207

Goldfarb, L., \& Treisman, A. (2011). Repetition blindness: The survival of the grouped. Psychonomic Bulletin \& Review, 18, 1042-1049. doi:10.3758/s13423-011-0135-4

Harris, C. L., \& Morris, A. L. (1998). Identity and similarity in repetition blindness: No cross-over interaction. Cognition, 81, 1-40. doi:10. 1016/S0010-0277(00)00138-4

Harris, C. L., \& Morris, A. L. (2000). Orthographic repetition blindness. Quarterly Journal of Experimental Psychology, 53A, 1039-1060. doi:10.1080/02724980050156281

Harris, C. L., \& Morris, A. L. (2004). Repetition blindness occurs in nonwords. Journal of Experimental Psychology: Human Perception and Performance, 30, 305-318. doi:10.1037/00961523.30.2.305

Jarvis, B. G. (2010). DirectRT (Version 2010) [Computer software]. New York, NY: Empirisoft Corp.

Kahneman, D., \& Treisman, A. (1992). The reviewing of object files: Object-specific integration of information. Cognitive Psychology, 24, 175-219. doi:10.1016/0010-0285(92)90007-O

Kahneman, D., Treisman, A., \& Burkell, J. (1983). The cost of visual filtering. Journal of Experimental Psychology: Human Perception and Performance, 9, 510-522. doi:10.1037/0096-1523.9.4.510

Kanwisher, N. G. (1987). Repetition blindness: Type recognition without token individuation. Cognition, 27, 117-143. doi:10.1016/00100277(87)90016-3
Kanwisher, N. (1991). Repetition blindness and illusory conjunctions: Errors in binding visual types with visual tokens. Journal of Experimental Psychology: Human Perception and Performance, 17, 404-421. doi:10.1037/0096-1523.17.2.404

Kanwisher, N., Driver, J., \& Machado, L. (1995). Spatial repetition blindness is modulated by selective attention to color or shape. Cognitive Psychology, 29, 303.337. doi:10.1006/coqp.1995.1017

Kanwisher, N. G., Kim, J. W., \& Wickens, T. D. (1996). Signal detection analyses of repetition blindness. Journal of Experimental Psychology: Human Perception and Performance, 22, 1249-1260. doi:10.1037/0096-1523.22.5.1249

Kanwisher, N., \& Potter, M. C. (1989). Repetition blindness: The effects of stimulus modality and spatial displacement. Memory \& Cognition, 17, 117-124. doi:10.3758/BF03197061

Kanwisher, N. G., \& Potter, M. C. (1990). Repetition blindness: Levels of processing. Journal of Experimental Psychology: Human Perception and Performance, 16, 30-47. doi:10.1037/0096-1523. 16.1.30

Lamy, D., Segal, H., \& Ruderman, L. (2006). Grouping does not require attention. Perception \& Psychophysics, 68, 17-31. doi:10.3758/ BF03193652

Luo, C. R., \& Caramazza, A. (1996). Temporal and spatial repetition blindness: Effects of presentation mode and repetition lag on the perception of repeated items. Journal of Experimental Psychology: Human Perception and Performance, 22, 95-113. doi:10.1037/ 0096-1523.22.1.95

Morris, A. L., \& Harris, C. L. (2002). Sentence context, word recognition, and repetition blindness. Journal of Experimental Psychology: Learning, Memory, and Cognition, 28, 962-982. doi:10.1037/ 0278-7393.28.5.962

Morris, A. L., \& Still, M. L. (2008). Now you see it, now you don't: Repetition blindness for nonwords. Journal of Experimental Psychology: Learning, Memory, and Cognition, 34, 146-166. doi: 10.1037/0278-7393.34.1.146

Mozer, M. C. (1989). Types and tokens in visual letter perception. Journal of Experimental Psychology: Human Perception and Performance, 15, 287-303. doi:10.1037/0096-1523.15.2.287

Neill, W. T., Neely, J. H., Hutchison, K. A., Kahan, T. A., \& VerWys, C. A. (2002). Repetition blindness, forward and backward. Journal of Experimental Psychology: Human Perception and Performance, 28, 137-149. doi:10.1037/0096-1523.28.1.137

Schendan, H. E., Kanwisher, N. G., \& Kutas, M. (1997). Early brain potentials link repetition blindness, priming and novelty detection. NeuroReport, 8, 1943-1948. doi:10.1097/00001756-19970526000030

Williams, M. C., \& Bologna, N. B. (1985). Perceptual grouping in good and poor readers. Perception \& Psychophysics, 38, 367-374. doi:10. 3758/BF03207165

Wong, K. F. E., \& Chen, H. (2009). Forward and backward repetition blindness in speed and accuracy. Journal of Experimental Psychology: Human Perception and Performance, 35, 778-786. doi:10.1037/a0013898 